\title{
Cardiovascular disease and the UN Millennium Development Goals: a serious concern
}

\author{
Valentin Fuster
}

According to the $\mathrm{WHO}$, total global deaths in 2005 amounted to 58 million, of which 35 million were from chronic diseases. Six of every 10 deaths were caused by chronic diseases, $80 \%$ of which occurred in countries with low and middle incomes. Cardiovascular disease (CVD), which accounted for 17.5 million deaths, is the leading cause of death globally. By contrast, among the infectious diseases, 2.8 million deaths were from HIV/AIDS, 1.6 million from tuberculosis, and 0.8 million from malaria; when combined, these three account for 3.3 times fewer deaths than those from CVD. Together, chronic diseases led to 6.4 times more mortality than these three infectious diseases combined. The only countries in which CVD is not the leading cause of death are those with the lowest incomes, most of which are in sub-Saharan Africa. Even in these countries, however, more than 12 million deaths were estimated to be from chronic diseases, versus 14 million from infectious diseases.

Despite numerous congresses and publications, one of the main reasons that CVD and other chronic diseases remain underrecognized and underfunded is the UN's Millennium Development Goals process. No pertinent representation of the chronic disease specialties is provided. CVD and chronic diseases, such as diabetes, chronic respiratory disease, and cancer, are being ignored by policy makers and development aid agencies, and limited funds mean limited action directed at prevention and control.

The UN's goals were designed to reduce poverty and promote health in developing countries, and should be reached by the year 2015 . Of the eight goals set, three were specifically targeted at health issues: reduction of childhood mortality by two-thirds relative to 1990; reduction of maternal mortality by three-quarters

\section{CVD and \\ chronic \\ diseases such \\ as diabetes, chronic \\ respiratory \\ disease and \\ cancer, are \\ being ignored \\ by policy \\ makers and \\ development \\ aid agencies}

$V$ Fuster is the Editorin-Chief of Nature

Clinical Practice

Cardiovascular

Medicine.

\section{Competing interests}

The author declared he has no competing interests.

www.nature.com/clinicalpractice doi:10.1038/ncpcardio0630 relative to 1990; and prevention of the spread of HIV/AIDS, malaria and other infectious diseases. The establishment of a linkage between health and economic development was applauded, but it is worrying that these health goals are so narrowly defined and fail to include preventive strategies for the chronic diseases, such as CVD. Chronic diseases are predicted to carry the highest economic cost and threaten the majority of the population in many low-income and middle-income countries.

Four compelling arguments can be made to include chronic diseases in the Millennium Development Goals process. First, analysis of the most recent global burden of disease data clearly reveals the predominance of CVD and other chronic diseases. Second, CVD affects many people of working age at high rates in countries with low and middle incomes, which clearly impacts economic growth. Third, health systems cannot be built vertically, disease by disease; by integrating chronic diseases, weak health systems can be strengthened. Finally, cost-effective policy, program, and treatment initiatives already exist for CVD and various other chronic diseases that could notably improve poverty and general health in many countries.

Interpretation of the Millennium Development Goals frequently seems to mean that health issues other than infectious diseases are ignored in poor countries. This situation must be rectified. In order to truly begin to reduce poverty, infectious and chronic diseases must be addressed together. The chronic disease community involved in the prevention and control of CVD in these vulnerable countries must be included in the Millennium Development Goals process at international and national levels. This recognition and support will help us all begin to tackle what threatens to be an overwhelming burden of disease. 